\title{
MSL_AR Toolkit: AR Authoring Tool with Interactive Features
}

\author{
Jinwook Shim, Jonghoon Seo, and Tack-don Han \\ Dept. of Computer Science, Yonsei University, \\ 134, Sinchon-dong, Seodaemun-gu, Seoul, Korea \\ \{jin99foryou, jonghoon. seo, hantack\} @msl.yonsei.ac.kr
}

\begin{abstract}
We describe an authoring tool for Augmented Reality (AR) contents. In recent years there have been a number of frameworks proposed for developing Augmented Reality (AR) applications. This paper describes an authoring tool for Augmented Reality (AR) application with interactive features. We developed the AR authoring tool which provides Interactive features that we can perform the education service project and participate it actively for the participating education service. In this paper, we describe MSL_AR Authoring tool process and two kinds of interactive features.
\end{abstract}

Keywords: Augmented Reality, Authoring, interaction.

\section{Introduction}

Continuous development in IT technology has been the main driving force of the new advancement and change throughout the whole society. The fields of education that requires high quality educational content service cannot be an exception. The educational environment of the future is expected to bring the learners to a participating education service where they actively join the service to show their individual creativity, out of the existing unidirectional educational service where the users only watch and listen to the given contents passively. Without realistic interface for wide participation, today`s educational environment, however, finds it hard to break-through the limitations of the existing educational pattern.

This paper focuses on a new way of education environment for user participation combining underlying Augmented Reality technologies which grant both educators and learners easy participation. We are suggesting the new type of participating educational environment by using Augmented Reality (AR) so that both educators and learners can participate easily.

We can provide guidelines about complicated sequence or the process of endanger experiment by using Augmented Reality (AR) with providing the virtual information which can be hard to observe and recognize the situation. In addition, we provide the educational effect with performing the tangible experiment by using the interaction technology. We are suggesting the Augmented Reality (AR) tool that provides two kinds of interactive features. 


\section{Related Work}

Current situation of AR Authoring Tools have too short history and lack of common application compare with 3ds MAX, MAYA. AR Authoring tool has a major ripple effect on technically yet. [1]

Table 1. Types of Authoring tools[1]

\begin{tabular}{|c|c|c|}
\hline & Programmers & Non-programmers \\
\hline \multirow{2}{*}{ Low level } & $\begin{array}{c}\text { ARToolKit } \\
\text { arTag[4] }\end{array}$ & $\begin{array}{c}\text { DART } \\
\text { ComposAR }\end{array}$ \\
\hline \multirow{2}{*}{ High level } & Studierstube & AMIRE \\
& osgART & MARS \\
\hline
\end{tabular}

ARToolKit[2] is an open-source library which provides computer vision based tracking of black square markers. However, to develop an AR application with ARToolKit requires further code for 3D model loading, interaction techniques, and other utility functions. This authoring tool is very simple structure and decoding algorithm. But, must load marker file and correlate for every marker to be detected. ARToolKit requires the developer to have c/c++ skills and needs to link with graphics and utility libraries. [2][3]

osgART is mainly based on the OpenSceneGraph framework, delivering them a pre-existing large choice of multimedia content but also the ability to import from professional designing tools (e.g. Maya, 3D Studio Max). The Tracking is mainly based on computer vision, using ARToolKit (extended also for supporting inertial tracking). [5][6][7]

One of the first AR authoring tools to support interactivity is DART [9], the Designer`s ARToolKit, which is a plug-in for the popular Macromedia Director software. The main aim of DART is to support application designers. DART is built to allow non-programmers to create AR experiences using the low-level AR services provided by the Director Xtras, and to integrate with existing Director behaviours and concepts. DART supports both visual programming and a scripting interface. [8][9]

composAR is a PC application that allows users to easily create AR scenes. It is based on osgART [5], and it is also a test beb for the use of scripting environments for OpenSceneGraph, ARToolKit and wxWidgets. [10][11]

AMIRE is an authoring tool for the efficient creation and modification of augmented reality applications. The AMIRE framework provides an interface to load and to replace a library at runtime and uses visual programming techniques to interactively develop AR applications. AMIRE is designed to allow content experts to easily build application without detailed knowledge about the underlying base technologies. [12][13] 


\section{MSL_AR Toolkit}

In this section we describe the prototype of a MSL_AR toolkit process. Our goal is to develop a low-level tool that will allow programmers to build AR contents with interactive features. The users can modify configure file that needs for interaction on AR content through GUI.

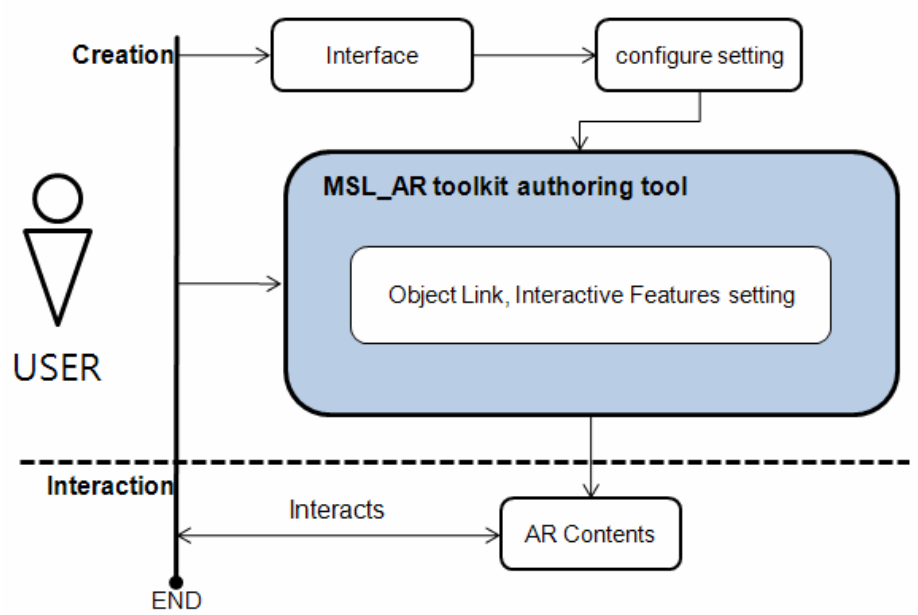

Fig. 1. MSL_AR toolkit authoring tool process

Figure 1 is a diagram of a MSL_AR toolkit authoring tool overall process. The first step is to input the information to create a simple AR content through User GUI. After that it creates AR content about entered information through main frame of MSL_AR toolkit.

The AR content that came out as the result of MSL_AR toolkit works with interactive feature which specified by the user.

\subsection{MSL_AR Toolkit Process}

MSL_AR toolkit authoring tool requires coding and scripting skills with programming knowledge. This authoring tool provides main function of engine and DLL library basically. Configure file determines markers` ID and the interaction methods, etc. Authoring tool that has been developed provides interaction that uses marker occlusion and marker merge methods. Figure 2 shows a flowchart of MSL_AR toolkit authoring tool.

User sets the configure file through interface. And then, user implements the MSL_AR toolkit, it creates the AR content by applying the configure file in the stage of preprocessor. When the process activates, it will find marker in the inputted video from camera and augments the object above the marker. It interacts by interactive feature with the information that user input. 


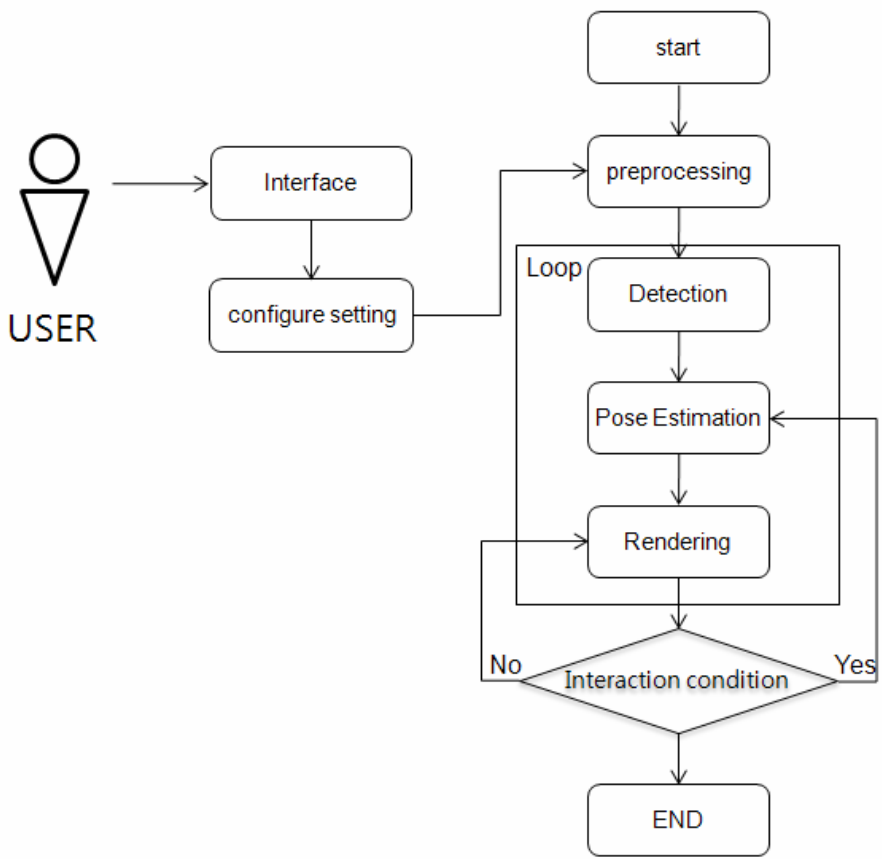

Fig. 2. MSL_AR toolkit flowchart

Figure 3 shows a main structure of MSL_AR toolkit authoring tool. "1class" of Figure 3 is a basic class to create AR content. " 4 tracking" part is a detection and pose estimation module of AR process.

The most important parts are "2initialize" and "5return" part of MSL_AR toolkit's main structure. The "2initialize" is a part that applies the configure file which user inputted through interface in process, and the "5return" is a part that shows the AR content with interactive feature.

\begin{tabular}{|c|c|c|}
\hline \multirow[t]{3}{*}{1 Class } & ARTK_MSL *artk = new ARTK_MSL(bool); & \\
\hline & & TRUE Capture mode enable \\
\hline & & FALSE Capture mode disable(DxShow based capture) \\
\hline \multirow[t]{2}{*}{ 2Initialize } & artk->initialize(const char"): & \\
\hline & & fileName Configure file name \\
\hline \multicolumn{2}{|c|}{3 Cpatured Img artk->capturelmage ; } & 1. TRUE \\
\hline \multirow[t]{4}{*}{4 Tracking } & artk $\rightarrow$ doTrack $\left(\right.$ char $\left.^{*}\right)$; & \\
\hline & & image 8 bits image \\
\hline & & NULL Captured Image tracking \\
\hline & & Iplimage OpenCV type \\
\hline \multicolumn{3}{|l|}{5 Return } \\
\hline & artk->configure & configure[i].visible==true \\
\hline
\end{tabular}

Fig. 3. MSL_AR toolkit authoring tool Main Structure 


\subsection{Configure Setting}

Figure 4 is a GUI picture that can register the object which is connected to marker and method that users can interact to marker in MSL_AR toolkit.

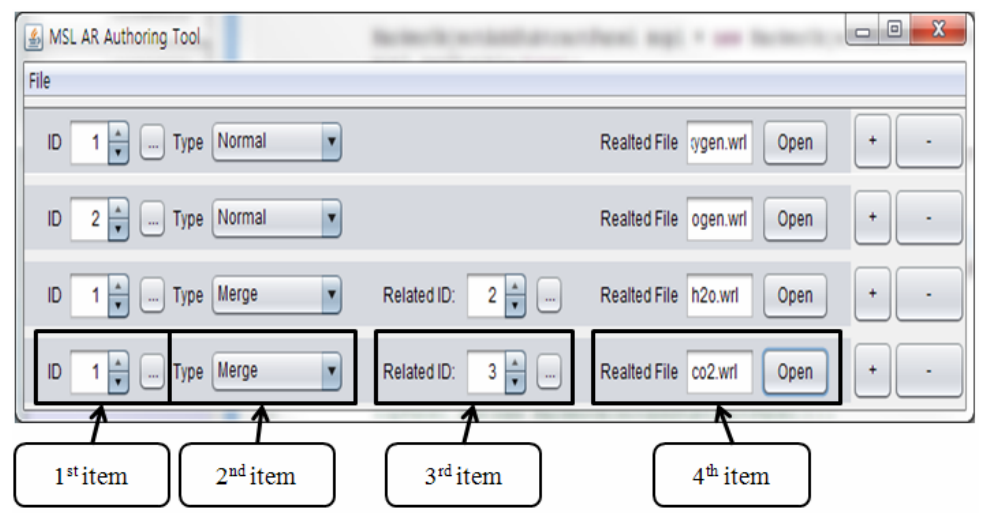

Fig. 4. MSL_AR toolkit configure set up GUI

The first item of Figure 4 designates marker ID. The second item designates interactive features. The third item designates the 3D object which is connected to marker. The fourth item reduces or adds ID of marker that will be used in contents. Through this interface, user can set up the AR content interaction comfortably.

Figure 5 shows the configure file content of MSL_AR toolkit that users can input through interface.

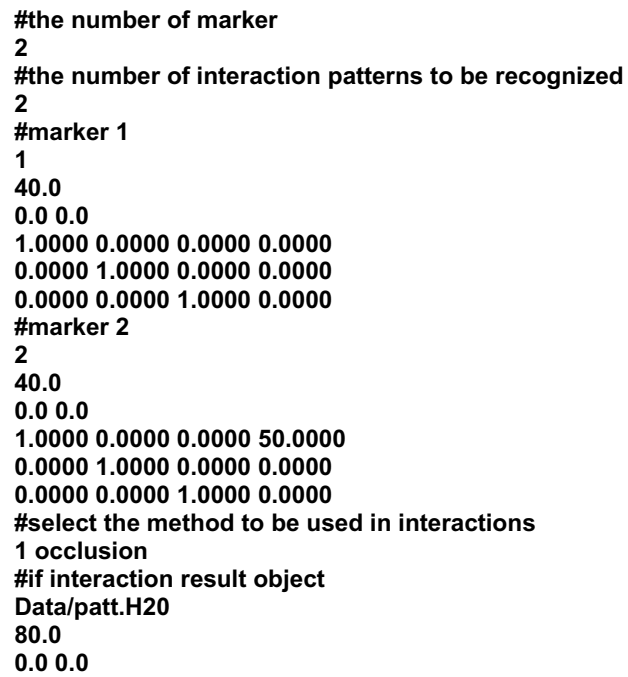

Fig. 5. Sample of MSL_AR toolkit configure file 
First of all, "the number of marker" means the number of marker that users will use. The second "the number of interaction patterns to be recognized" means the number of markers that will be used in interaction. The third "marker 1" and "marker 2" mean marker ID, size of marker, relative coordinates of marker. The fourth "select the method to be used in interactions" means interactive feature that will be used in interaction. The fifth "if interaction result object" means the information of object that appears when interaction happens between markers.

\subsection{Interactive Features}

MSL_AR toolkit authoring tool provides two kinds of interactive features. One of them is an occlusion method that performs when marker is covered. And the other is a merge method that performs when two markers are located closely.

Occlusion. Figure 6 shows an occlusion interactive feature of MSL_AR toolkit authoring tool when marker was covered by user`s finger. The left figure of Figure 6 shows two markers that registered by user. The right figure shows occlusion interactive feature of MSL_AR toolkit. One marker augments the square object as a result of interaction on another marker when a marker is covered by user's finger.
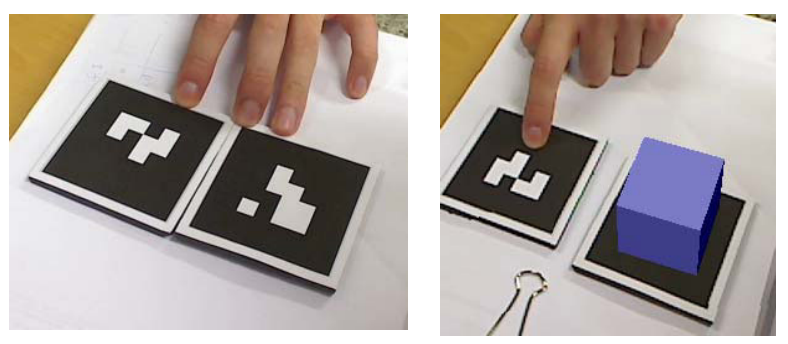

Fig. 6. Occlusion Interactive feature

User selects the GUI $2^{\text {nd }}$ item of Figure 4 as "occlusion" and selects the marker that will augment the object on the $3^{\text {rd }}$ item. $4^{\text {th }}$ item of GUI is an object that will augment above the marker.

After finishing the inputting the GUI, these data are entered into "\#select the method to be used in interactions" and "\#if interaction result object" of Figure 5's configure file.

Merge. Figure 7 shows a merge interactive feature of MSL_AR toolkit authoring tool when two markers are located closely. The right figure is a result that is merge interactive feature of MSL_AR toolkit. When two markers merged, "Oxygen" marker augments the $\mathrm{H} 20$ molecule object as a result of interaction. 

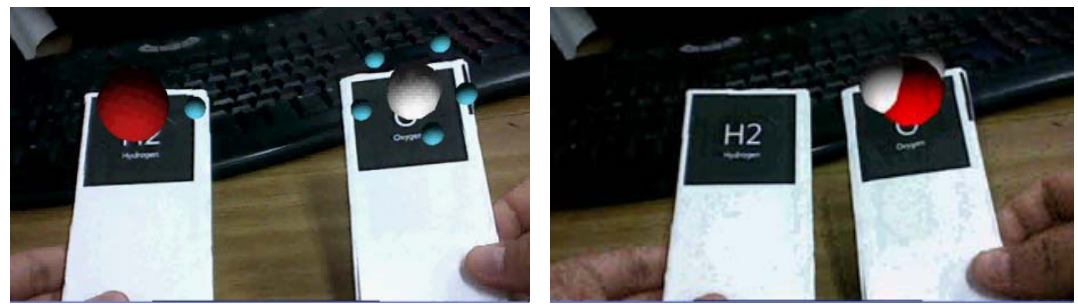

Fig. 7. Merge Interactive feature

User selects the GUI $2^{\text {nd }}$ item of Figure 4 as "merge" and the rest of the item are selected as above.

\section{Conclusion}

The existing limitation of Augmented Reality authoring tool is fixed and provided restricted contents. Thus, there is no more additional information. The function of Augmented Reality based offers additional virtual information; that is actually difficult to observe, recognizes the situation and provides guideline about experimental procedure or order, etc. Also through interaction technology, MSL_AR toolkit increases the educational effectiveness and can be performed effectual, tangible experiments using marker.

Non-programmer can produce contents easily by developing improved GUI from future work and has to study interaction method which is a variety of location-based marker. And much more active and efficient to produce AR contents, we have to make study additional interactive features such as keyboard listener and will be able to control phase of contents.

Acknowledgement. This work (2010-0027654) was supported by Mid-career Researcher Program through NRF grant funded by the MEST.

\section{References}

1. Wang, Y., Langlotz, T., Bilinghurst, M., Bell, T.: An Authring Tool for Mobile Phone AR Environments. In: NZCSRSC 2009 (2009)

2. Azuma, R.: A survey of Augmented Reality. Presence: Teloperators and Virtual Environments 6(4), 355-385 (1997)

3. http://www.hitl.washington. edu/artoolkit/

4. Fiala, M.: ARTag, a fiducial marker system using digital techniques. In: IEEE Computer Society Conference on Computer Vision and Pattern Recognition, CVPR 2005, vol. 592, pp. 590-596 (2005)

5. http://www.cc.gatech.edu/ael/resources/osgar.html

6. http://www.artoolworks. com/community/osgart/

7. Grasset, R., Looser, J., Billinghurst, M.: OSGARToolKit: tangible + transitional 3D collaborative mixed reality framework. In: Proceedings of the 2005 International Conference on Augmented Tele-Existence, ICAT 2005, Christchurch, New Zealand, December 05-08, vol. 157, pp. 257-258. ACM, New York (2005) 
8. http: / / www.gvu.gatech.edu/dart/

9. MacIntyre, B., Gandy, M., Dow, S., Bolter, J.D.: DART: a toolkit for rapid design exploration of augmented reality experiences. In: Marks, J. (ed.) SIGGRAPH 2005, ACM SIGGRAPH 2005 Papers, Los Angeles, California, July 31-August 04, pp. 932-932. ACM, New York (2005)

10. http://www.hitlabnz.org/wiki/ComposAR

11. Dongpyo, H., Looser, J., Seichter, H., Billinghurst, M., Woontack, W.: A Sensor-Based Interaction for Ubiquitous Virtual Reality Systems. In: International Symposium on Ubiquitous Virtual Reality, ISUVR 2008, pp. 75-78 (2008)

12. http: / / www . amire.net/

13. Grimm, P., Haller, M., Paelke, V., Reinhold, S., Reimann, C., Zauner, R.: AMIRE authoring mixed reality. In: The First IEEE International Workshop Augmented Reality Toolkit, p. 2 (2002) 